\title{
High rates of severe disease and death due to SARS-CoV-2 infection in rheumatic disease patients treated with rituximab: a descriptive study
}

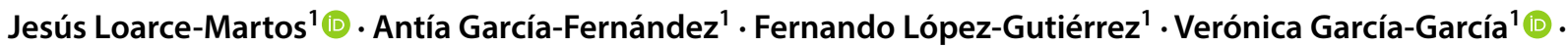 \\ Laura Calvo-Sanz ${ }^{1} \cdot$ Iván del Bosque-Granero ${ }^{1} \cdot$ M. Andreína Terán-Tinedo ${ }^{1}$ - Alina Boteanu ${ }^{1}$. \\ Javier Bachiller-Corral ${ }^{1}$ [D $\cdot$ Mónica Vázquez-Díaz ${ }^{1}$
}

Received: 22 July 2020 / Accepted: 31 August 2020 / Published online: 18 September 2020

(c) Springer-Verlag GmbH Germany, part of Springer Nature 2020

\begin{abstract}
The objective of this study is to describe the characteristics and outcomes of rheumatic and musculoskeletal disease (RMD) patients who were treated with rituximab and had suspected or confirmed severe acute respiratory syndrome coronavirus 2 (SARS-CoV-2) infection. In this descriptive study, RMD patients who were treated with rituximab in the last 12 months at the Rheumatology Department of our hospital were screened for SARS-CoV-2 infection via telephone interview and a comprehensive review of clinical health records (01/02/2020-26/05/2020). Those with probable or confirmed SARS-CoV-2 infection were included. In total, 76 patients were screened. Of these, 13 (17.1\%) had suspected or confirmed SARS-CoV-2 infection. With regard to these 13 patients, the median age at coronavirus disease (COVID-19) diagnosis was 68 years (range 28-76 years) and 8 (61.5\%) were female. Five patients had rheumatoid arthritis, three had systemic vasculitis, two had Sjögren syndrome, and two had systemic lupus erythematosus. Additionally, seven patients (53.8\%) had pulmonary involvement secondary to RMD. Eight patients $(61.5 \%)$ developed severe disease leading to hospitalization, and seven developed bilateral pneumonia and respiratory insufficiency. Of the eight hospitalized patients, five (62.5\%) fulfilled the acute respiratory distress syndrome criteria and three developed a critical disease and died. Our cohort had a high rate of severe disease requiring hospitalization (61.5\%), with bilateral pneumonia and hyperinflammation leading to a high mortality rate (23.1\%). Treatment with rituximab should be considered a possible risk factor for unfavorable outcomes in COVID-19 patients with RMD. However, further study is required to confirm this association.
\end{abstract}

Keywords Rheumatic diseases $\cdot$ Rituximab $\cdot$ SARS-CoV-2 $\cdot$ COVID-19

\section{Introduction}

Severe acute respiratory syndrome coronavirus 2 (SARSCoV-2) infection was first reported in Wuhan, China, in December 2019. The spread of SARS-CoV-2 infection was declared a pandemic in March 2020 by the World Health Organization with 800,906 deaths among 2,057,288

Jesús Loarce-Martos and Antía García-Fernández have equal contribution.

Jesús Loarce-Martos

jesus.loarce@gmail.com

1 Rheumatology Department, Hospital Universitario Ramón y Cajal, Carretera de Colmenar Viejo, 9, 1 km, 28043 Madrid, Spain confirmed cases, which accounted for $3.89 \%$ of mortality among infected individuals [1]. Madrid, Spain, is a highimpact area in terms of SARS-CoV-2 infection, with official data showing more than 74,886 infected people and 8,451 infection-related deaths to date (24/08/2020) [2]. SARS$\mathrm{CoV}-2$ is an enveloped, single-stranded RNA virus and a member of $\beta$-coronaviruses, such as severe acute respiratory syndrome coronavirus (SARS-CoV) and Middle East respiratory syndrome coronavirus. Similar to SARS-CoV, SARS-CoV-2 enters human cells using angiotensin-converting enzyme 2 and causes coronavirus disease (COVID-19). COVID-19 severity ranges from asymptomatic to severe, and the disease can result in acute respiratory distress syndrome (ARDS) and cytokine storm, which have led to death in $3-10 \%$ of the infected population. 
Patients with rheumatic and musculoskeletal diseases (RMDs) are at high risk for infections related to their disease, comorbidities, or immunosuppressive treatments [3], but recent studies regarding COVID-19 did not support higher mortality in these patients [4-7]. However, there are few data regarding specific risk factors for unfavorable outcomes, such as specific diseases or immunosuppressive drugs [5]. Further, some reports have suggested the worst prognosis for RMD patients who were treated with rituximab and developed SARS-CoV-2 infection [8,9]. Our objective was to describe the clinical characteristics and outcomes of our cohort of RMD patients who were treated with rituximab and had suspected or confirmed SARS-CoV-2 infection.

\section{Methods}

In this descriptive study, RMD patients who were treated with rituximab in the last 12 months at the Rheumatology Department of our hospital (Hospital Universitario Ramón y Cajal, Madrid, Spain) were screened for SARS-CoV-2 infection via telephone interview and a comprehensive review of clinical health records (02/01/2020-26/05/2020). Those with probable or confirmed SARS-CoV-2 infection were included. There were no exclusion criteria.

SARS-CoV-2 infections were confirmed if patient's nasopharyngeal swabs were positive for SARS-CoV-2, as determined via real-time reverse transcription polymerase chain reaction (RT-PCR) and/or characteristic bilateral infiltrates were found on chest radiographs/computerized tomography (CT) scans. A probable SARS-CoV-2 infection was defined as the presence of two or more symptoms related to COVID19 (fever, dry cough, dyspnea, odynophagia, myalgia, anosmia, or ageusia) when other causes were excluded. TaqMan 2019-nCoV Assay Kit v1 (Thermo Fisher Scientific Inc., Franklin, MA, USA) was used to detect viral RNA. It has high efficiency, similar to other commercially available RTPCR tests [10].

Hyperinflammation was defined in accordance with the ongoing clinical trials and recent studies (lymphocyte count $<1000$ cells $/ \mathrm{mL}$ and two of the following criteria: serum ferritin level $>500 \mathrm{ng} / \mathrm{mL}$, lactate dehydrogenase level $>300 \mathrm{U} / \mathrm{L}$, and D-dimer level $>1000 \mathrm{ng} / \mathrm{m}$ ), and was evaluated within the first 7 days of hospital admission [11].

The current study is a sub-analysis of a larger study focused in SARS-CoV-2 infection in RMD patients (study number: 136/20), and was approved by the local ethics committee of our hospital (comité de ética de la investigación con medicamentos del Hospital Universitario Ramón y Cajal) on 05/05/2020. All patients provided informed consent to participate and for publication prior to their inclusion.
Categorical variables are presented as numbers and percentages. Continuous variables are expressed as median (interquartile range [IQR] or range).

\section{Results}

\section{Patient characteristics}

We screened a total of 76 patients. Of these 76 patients, 63 (82.9\%) did not exhibit COVID-19 symptoms or signs of suspected SARS-CoV-2 infection, and 13 (17.1\%) had suspected or confirmed SARS-CoV-2 infection. The baseline characteristics of these 13 patients are described in Table 1. The median age at COVID-19 diagnosis was 68 years (range 28-76 years) and eight (61.5\%) were female. Further, rheumatoid arthritis (RA) was the most frequent RMD (6/13, $46.2 \%$ ) observed in patients with suspected or confirmed SARS-CoV-2 infection, followed by systemic vasculitis (3/13, 23\%), Sjögren syndrome $(2 / 13,15.4 \%)$, and systemic lupus erythematosus (SLE) $(2 / 13,15.4 \%)$. With regard to other immunosuppressive treatments prescribed for their RMD, six patients were treated with methotrexate, and one patient was treated with hydroxychloroquine (HCQ). Further, of the 13 suspected and confirmed SARS-CoV-2 infection patients, 7 (53.8\%) were on low-dose corticosteroid treatment ( $\leq 10 \mathrm{mg} /$ day). Moreover, the main comorbidities observed in this group were arterial hypertension (61.2\%), dyslipidemia (46.2\%), chronic obstructive pulmonary disease (COPD) or asthma (46.2\%), cardiovascular disease (23.1\%), and diabetes mellitus (7.7\%). Additionally, pulmonary involvement secondary to RMD was present in 7 of the 13 patients (53.8\%), and interstitial lung disease (ILD) was observed in 3 of those 7 patients (42.9\%).

With regard to their RMD activity at COVID-19 diagnosis, $76.9 \%$ of patients were in remission, $15.4 \%$ had low disease activity, and $7.7 \%$ had active disease, despite receiving treatment.

\section{Diagnosis and symptoms}

The main characteristics of SARS-CoV-2 infection and treatment are summarized in Table 2. Among the 13 patients with suspected or confirmed SARS-CoV-2 infection, 8 (61.5\%) had confirmed infections and $5(38.5 \%)$ had suspected infections. The most frequently reported COVID-19 symptoms were fever $(61.5 \%)$, asthenia (61.5\%), cough $(61.5 \%)$, and dyspnea $(61.5 \%)$. Diarrhea was present in $38.5 \%$ of suspected and confirmed COVID-19 patients. Anosmia and ageusia were only reported in $15.4 \%$ and $7.7 \%$ of such patients, respectively.

With regard to hospitalization, 8 of the 13 patients $(61.5 \%)$ were treated as inpatients and hospitalized for a 


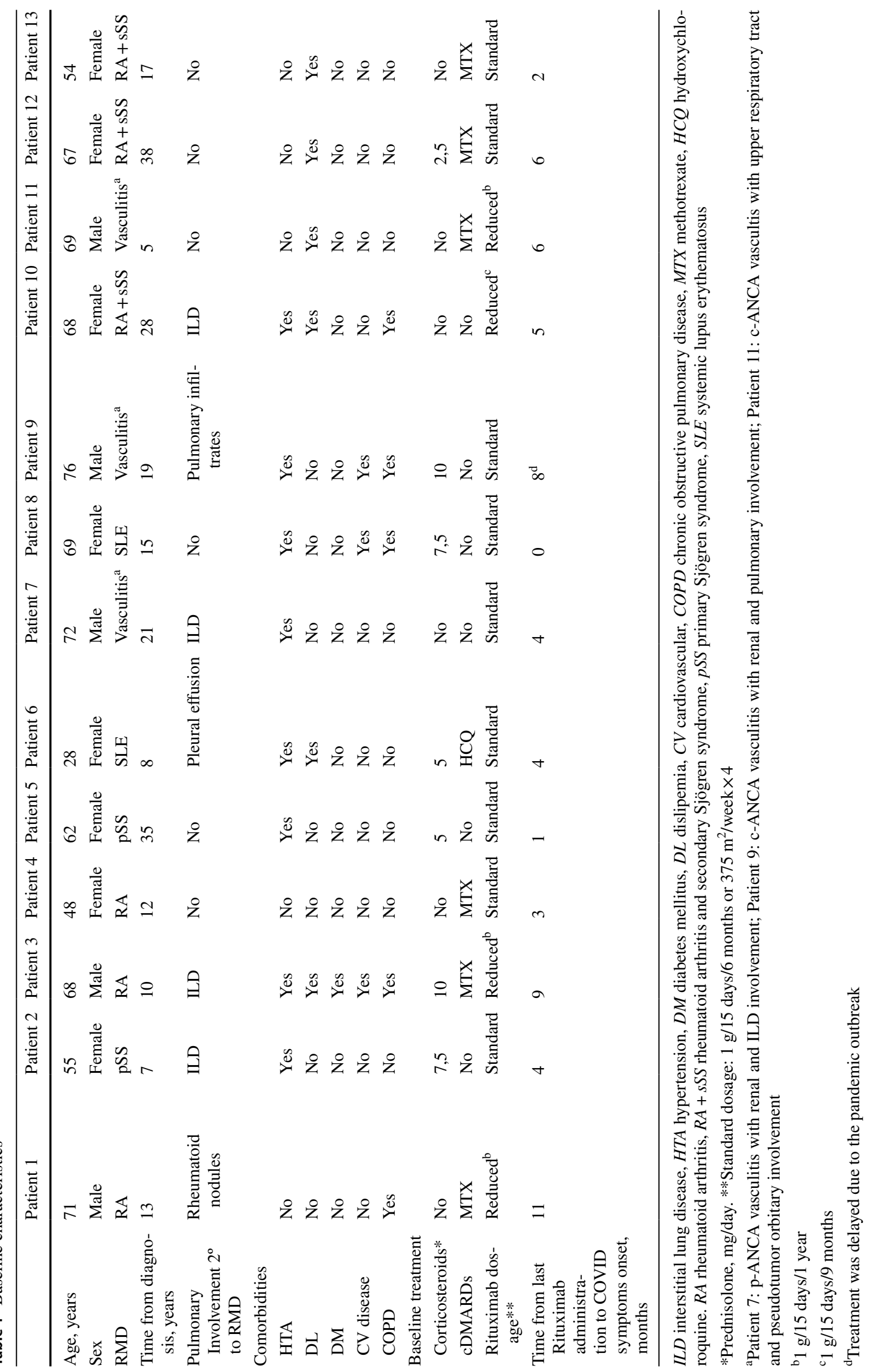




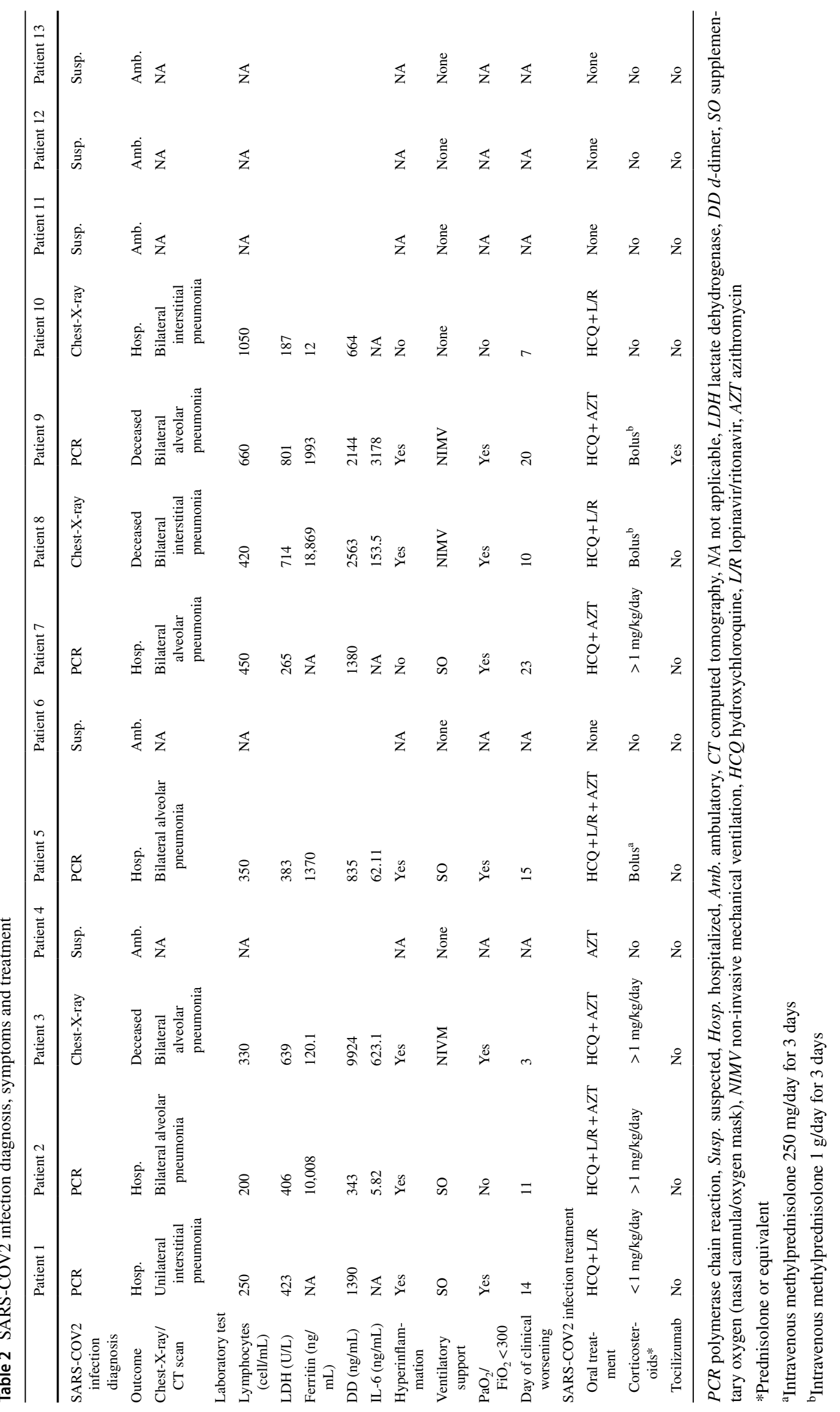


median of 10 days (range 4-29 days). All eight patients had confirmed SARS-CoV-2 infections. Furthermore, laboratory tests were only performed for hospitalized patients. Of these eight patients, six $(75 \%)$ presented with hyperinflammation. Interleukin-6 levels were measured in five patients and found to be elevated in four (80\%) of them, as reported in Table 2 . Chest radiographs, which were only available for inpatients, showed that seven $(87.5 \%)$ inpatients had bilateral pneumonia. Repeated chest X-rays or CT scans were performed after a median of 6 days (range 3-11 days) for these seven inpatients and showed that three patients $(42.8 \%)$ exhibited radiological improvement, one (14.2\%) remained stable, and three $(42.8 \%)$ worsened.

\section{Treatment}

All ambulatory patients $(n=5)$ maintained their treatment with conventional disease-modifying antirheumatic drugs (DMARDs). Additionally, one patient received azithromycin, but no other treatment was prescribed.

All hospitalized patients suspended their conventional DMARD treatment at admission and were prescribed HCQ, along with azithromycin or lopinavir/ritonavir. Corticosteroids were only prescribed for hospitalized patients with severe disease, as determined by the treating clinician. Intravenous methylprednisolone was prescribed to seven of the eight hospitalized patients (87.5\%). Of these seven patients, two $(28.5 \%)$ received $1 \mathrm{~g}$ of methylprednisolone per day for 3 days, one $(14.3 \%)$ received $250 \mathrm{mg}$ of methylprednisolone per day for 3 days, three $(42.9 \%)$ received $>1 \mathrm{mg} / \mathrm{kg}$ of methylprednisolone per day for a median of 8 days, and one (14.3\%) received $<1 \mathrm{mg} / \mathrm{kg}$ of methylprednisolone per day for 7 days.

\section{Outcomes}

All ambulatory patients $(n=5)$ exhibited favorable outcomes.

All eight hospitalized patients experienced clinical worsening after a median of 12.5 days (range 3-23 days; $62.5 \%$ of patients worsened after $>10$ days from symptom onset). Respiratory insufficiency requiring the use of supplementary oxygen was observed in seven hospitalized patients (87.5\%). Of these seven patients, three $(42.9 \%)$ required non-invasive mechanical ventilation (NIVM). Additionally, five of the eight hospitalized patients (62.5\%) fulfilled the ARDS criteria $\left(\mathrm{PaO}_{2} / \mathrm{FiO}_{2}<300\right.$ and bilateral pneumonia).

Furthermore, three of the thirteen patients with suspected or confirmed SARS-CoV-2 infection $(23.1 \%)$ died due to severe ARDS, despite intensive treatment and NIVM. All patients who died had three or more comorbidities and two had pulmonary involvement secondary to RMD. With regard to other complications, an RA patient (Patient 3) who was admitted to our hospital due to multiple osteoporotic fractures developed a pulmonary embolism, despite taking prophylactic heparin. Patient 8, a woman diagnosed with SLE secondary to Evans syndrome and COPD which was treated with chronic supplementary oxygen, developed a secondary Pseudomonas aeruginosa infection.

\section{Discussion}

To our knowledge, ours is the first study that describes a cohort of RMD patients who were treated with rituximab and were determined or suspected of having COVID-19 after comprehensive screening. We report a relatively high rate of infection among rituximab-treated RMD patients $(13 / 76,17 \%)$. Additionally, a large portion of our cohort of infected patients $(n=13)$ was frequently hospitalized $(61.5 \%)$ and had a high mortality rate $(23.1 \%)$. Few studies have described SARS-CoV-2 infection among RMD patients and those that undergo immunosuppressive treatments, but all of them have reported lower hospitalization and death rates compared to our patients, with mortality ranging between 0 and 10\% [4-6, 11-13]. However, given the small number of patients in these studies, very few have analyzed specific risk factors related to immunosuppressive therapies, and most of them have reported higher hospitalization rates for patients receiving chronic glucocorticoids compared to those not receiving them [4, 6 , 8]. Interestingly, one of these studies reported an inverse relationship between anti-TNF therapy and hospitalization [6]. Nevertheless, specific information about rituximab is lacking in most of these studies. Favalli et al. [14] reported on a cohort in which one COVID-19 patient was treated with rituximab. This patient, a 32-year-old woman with systemic sclerosis and pulmonary involvement, died after requiring mechanical ventilation and treatment with tocilizumab. Furthermore, the BIOBADASER cohort (from a multicenter Spanish registry assessing patients treated with biological or synthetic DMARDs) had two SARS-CoV-2-infected patients who were treated with rituximab, one of whom died (91 years old, hypertension, systemic vasculitis) [12]. Another study from Madrid, Spain recently reported that their entire cohort of seven RMD patients who were treated with rituximab developed SARS-CoV-2 infections requiring hospitalization, and one (14.2\%) died due to respiratory failure [8]. We also found 10 COVID-19 patients with RMD who were treated with rituximab in the current literature. These 10 patients were from various case reports and case series. Of these 10 patients, 8 had bilateral pneumonia requiring hospitalization and supplementary oxygen and three died [9, 15-20]. All these reports suggest a possible association between 
rituximab treatment for RMDs and poor prognoses for SARS-CoV-2 infected patients, but no study to date has analyzed the risk of SARS-CoV-2 infection and prognosis of such patients.

Rituximab is a chimeric monoclonal anti-CD20 antibody that has been approved for treating RA that is unresponsive to initial biological DMARD treatment, microscopic polyangiitis, and granulomatosis with polyangiitis. It is also widely used for treating other systemic diseases, such as SLE, Sjögren syndrome, systemic sclerosis, and idiopathic inflammatory myopathies. Several studies have described the effect of rituximab on the B cell cytokine network, mainly on B effector and B regulator cells, with a secondary influence on T helper cell response [21, 22]. Some studies have reported a higher risk of infection in RA patients treated with rituximab than those treated with other biologic DMARDs [23, 24] but these data come from registries and retrospective studies, which may contain bias due to their observational designs. The effects of rituximab on the immune system response to SARS-CoV-2 infection have yet to be elucidated.

Notably, one of our study's strengths is the fact that it is the first study in which all rituximab-treated patients for their RMD from the same hospital were clinically screened for SARS-COV-2 infection and had their disease characteristics and outcomes thoroughly reported on. This is relevant given that the possible association between rituximab treatment and poor prognoses was made based on case reports or case series, and information about the total cohort of patients treated with rituximab from which they come from is lacking.

However, our study's main limitation is that our cohort was assessed retrospectively and it does not have a control group. Our cohort exhibited comorbidities such as hypertension COPD/asthma and ILD which are associated with poor prognoses [6] and may have influenced the results. Besides, our cohort size was relatively small and comparisons between groups could not be performed.

In conclusion, ours is the first systematic report on SARSCoV-2 infection among RMD patients who were treated with rituximab. This cohort exhibited a high rate of severe infection requiring hospitalization $(61.5 \%)$, with bilateral pneumonia and hyperinflammation leading to a high mortality rate $(23.1 \%)$. Therefore, treatment with rituximab should be considered a possible risk factor for unfavorable outcomes in COVID-19 patients with RMD. However, further study is necessary to confirm this association.

Author contributions All authors contributed to the study conception and design. Material preparation, data collection and analysis were performed by all authors. The first draft of the manuscript was written by JLM and AGF, and all authors commented on previous versions of the manuscript. All authors read and approved the final manuscript.
Funding No specific funding was received from any bodies in the public, commercial or not-for-profit sectors to carry out the work described in this article.

\section{Compliance with ethical standards}

Conflict of interest Dr. Loarce-Martos reports personal fees from Celgene S.L.U., outside the submitted work; Dr. García-Fernández has nothing to disclose; Dr. López-Gutiérrez has nothing to disclose; Dr. García-García has nothing to disclose; Dr. Calvo-Sanz has nothing to disclose; Dr. del Bosque-Granero has nothing to disclose; Dr. TeránTinedo has nothing to disclose; Dr. Boteanu has nothing to disclose; Dr. Bachiller-Corral has nothing to disclose; Dr. Vázquez-Díaz reports personal fees and non-financial support from Sandoz, personal fees and non-financial support from Pfizer, personal fees and non-financial support from Merck Sharp and Dohme, outside the submitted work.

Ethical approval The current study is a sub-analysis of a larger study focused in SARS-CoV-2 infection in RMD patients (study number: $136 / 20$ ), and was approved by the local ethics committee of our hospital (comité de ética de la investigación con medicamentos del Hospital Universitario Ramón y Cajal) on 05/05/2020. All patients provided informed consent to participate and for publication prior to their inclusion.

\section{References}

1. WHO Coronavirus Disease (COVID-19) Dashboard I WHO Coronavirus Disease (COVID-19) Dashboard. https://covid19.who.int/. Accessed 24 Aug 2020

2. Datos COVID-19 Comunidad de Madrid. https://www.comun idad.madrid/uploads/mapas_covid-19/index.html. Accessed 24 Aug 2020

3. Hsu CY, Ko CH, Wang JL et al (2019) Comparing the burdens of opportunistic infections among patients with systemic rheumatic diseases: a nationally representative cohort study. Arthritis Res Ther. https://doi.org/10.1186/s13075-019-1997-5

4. Haberman R, Axelrad J, Chen A et al (2020) Covid-19 in immunemediated inflammatory diseases-case series from New York. N Engl J Med. https://doi.org/10.1056/nejmc2009567

5. Michelena X, Borrell H, López-Corbeto M et al (2020) Incidence of COVID-19 in a cohort of adult and paediatric patients with rheumatic diseases treated with targeted biologic and synthetic disease-modifying anti-rheumatic drugs. Semin Arthritis Rheum. https://doi.org/10.1016/j.semarthrit.2020.05.001

6. Gianfrancesco M, Hyrich KL, Al-Adely S et al (2020) Characteristics associated with hospitalisation for COVID-19 in people with rheumatic disease: data from the COVID-19 Global Rheumatology Alliance physician-reported registry. Ann Rheum Dis 79:859-866. https://doi.org/10.1136/annrheumdis-2020-217871

7. D'Silva KM, Serling-Boyd N, Wallwork R et al (2020) Clinical characteristics and outcomes of patients with coronavirus disease 2019 (COVID-19) and rheumatic disease: a comparative cohort study from a US "hot spot". Ann Rheum Dis. https://doi. org/10.1136/annrheumdis-2020-217888

8. Nuño L, Novella Navarro M, Bonilla G et al (2020) Clinical course, severity and mortality in a cohort of patients with COVID-19 with rheumatic diseases. Ann Rheum Dis. https://doi. org/10.1136/annrheumdis-2020-218054

9. Schulze-Koops H, Krueger K, Vallbracht I et al (2020) Increased risk for severe COVID-19 in patients with inflammatory rheumatic 
diseases treated with rituximab. Ann Rheum Dis. https://doi. org/10.1136/annrheumdis-2020-218075

10. Iglói Z, leven M, Abdel-Karem Abou-Nouar Z, et al (2020) Comparison of commercial realtime reverse transcription PCR assays for the detection of SARS-CoV-2. J Clin Virol 129:10-12. https ://doi.org/10.1016/j.jcv.2020.104510

11. Fredi M, Cavazzana I, Moschetti L et al (2020) COVID-19 in patients with rheumatic diseases in northern Italy: a singlecentre observational and case-control study. Lancet Rheumatol 2(9):e549-e556. https://doi.org/10.1016/S2665-9913(20)30169-7

12. Sanchez-Piedra C, Diaz-Torne C, Manero J et al (2020) Clinical features and outcomes of COVID-19 in patients with rheumatic diseases treated with biological and synthetic targeted therapies. Ann Rheum Dis. https://doi.org/10.1136/annrheumdis-2020217948

13. Monti S, Balduzzi S, Delvino P et al (2020) Clinical course of COVID-19 in a series of patients with chronic arthritis treated with immunosuppressive targeted therapies. Ann Rheum Dis 79:667-668

14. Favalli EG, Agape E, Caporali R (2020) Incidence and clinical course of COVID-19 in patients with connective tissue diseases: a descriptive observational analysis. J. Rheumatol 47(8):1296. https ://doi.org/10.3899/jrheum.200507

15. Guilpain P, Le Bihan C, Foulongne V et al (2020) Rituximab for granulomatosis with polyangiitis in the pandemic of COVID-19: lessons from a case with severe pneumonia. Ann Rheum Dis. https ://doi.org/10.1136/annrheumdis-2020-217549

16. Avouac J, Airó P, Carlier N et al (2020) Severe COVID-19-associated pneumonia in 3 patients with systemic sclerosis treated with rituximab. Ann Rheum Dis. https://doi.org/10.1136/annrheumdi s-2020-217864

17. Hakroush S, Franz J, Larsen J et al (2020) Repeated false-negative tests delayed diagnosis of COVID-19 in a case with granulomatosis with polyangiitis under maintenance therapy with rituximab and concomitant influenza pneumonia. Ann Rheum Dis. https:// doi.org/10.1136/annrheumdis-2020-218491
18. Benucci M, Quartuccio L, Li Gobbi F et al (2020) Persistence of rT-PCR-SARS-CoV-2 infection and delayed serological response, as a possible effect of rituximab according to the hypothesis of Schulze-Koops et al. Ann Rheum Dis. https://doi.org/10.1136/ annrheumdis-2020-218590

19. Suárez-Díaz S, Morán-Castaño C, Coto-Hernández R et al (2020) Mild COVID-19 in ANCA-associated vasculitis treated with rituximab. Ann Rheum Dis. https://doi.org/10.1136/annrheumdi s-2020-218246

20. Fallet B, Kyburz D, Walker UA (2020) Mild course of COVID19 and spontaneous virus clearance in a patient with depleted peripheral blood B cells due to rituximab treatment. Arthritis Rheumatol. https://doi.org/10.1002/art.41380

21. Luu VP, Vazquez MI, Zlotnik A (2014) B cells participate in tolerance and autoimmunity through cytokine production. Autoimmunity $47: 1-12$

22. Pateinakis P, Pyrpasopoulou A (2014) CD20+ B cell depletion in systemic autoimmune diseases: common mechanism of inhibition or disease-specific effect on humoral immunity? Biomed Res Int. https://doi.org/10.1155/2014/973609

23. Grøn KL, Arkema EV, Glintborg B et al (2019) Risk of serious infections in patients with rheumatoid arthritis treated in routine care with abatacept, rituximab and tocilizumab in Denmark and Sweden. Ann Rheum Dis 78:320-327. https://doi.org/10.1136/ annrheumdis-2018-214326

24. Yun H, Xie F, Delzell E et al (2016) Comparative risk of hospitalized infection associated with biologic agents in rheumatoid arthritis patients enrolled in medicare. Arthritis Rheumatol 68:5666. https://doi.org/10.1002/art.39399

Publisher's Note Springer Nature remains neutral with regard to jurisdictional claims in published maps and institutional affiliations. 